\title{
Identification of the Sequence of Steps Intrinsic to Spheromak Formation
}

\author{
P.M. Bellan, S. You, and G. S. Yun \\ California Institute of Technology \\ Pasadena, CA 91125, USA
}

\begin{abstract}
A planar coaxial electrostatic helicity source is used for studying the relaxation process intrinsic to spheromak formation. Experimental observations reveal that spheromak formation involves: (1) breakdown and creation of a number of distinct, arched, filamentary, plasma-filled flux loops that span from cathode to anode gas nozzles, (2) merging of these loops to form a central column, (3) jet-like expansion of the central column, (4) kink instability of the central column, (5) conversion of toroidal flux to poloidal flux by the kink instability. Steps 1 and 3 indicate that spheromak formation involves an MHD pumping of plasma from the gas nozzles into the magnetic flux tube linking the nozzles. In order to measure this pumping, the gas puffing system has been modified to permit simultaneous injection of different gas species into the two ends of the flux tube linking the wall. Gated CCD cameras with narrow-band optical filters are used to track the pumped flows.
\end{abstract}

Keywords: spheromak, magnetohydrodynamics, kink, jet

PACS: 52.55.Ip, 52.35.Py, 52.55.Wq, 52.59.Dk

\section{INTRODUCTION}

Spheromaks are toroidal MHD equilibria incorporating nested poloidal magnetic flux surfaces suitable for confining a fusion plasma. Unlike tokamaks, spheromaks are a self-organizing, minimum-energy state and so, in principle, require little hardware beyond that needed to inject magnetic helicity into a flux conserver. The minimum-energy final state of the self-organizing process is predicted by the WoltjerTaylor model and consists of a toroidal equilibrium having equal poloidal and toroidal magnetic field energies. Practical spheromak formation can be achieved using either electrostatic or inductive helicity sources. These inject helicity-laden plasma having unequal amounts of poloidal and toroidal magnetic field energy. After injection, MHD instabilities develop and re-arrange the poloidal and toroidal fluxes in such a way as to relax the configuration towards a minimum energy state while conserving magnetic helicity (roughly the product of the toroidal and poloidal fluxes).

A planar coaxial electrostatic helicity source has been built [1]-[4] to reveal the details of this relaxation process. This source, sketched in Fig. 1, consists of a disk electrode surrounded by a coaxial annulus electrode. A coil produces magnetic field spanning the two electrodes. Like conventional coaxial helicity sources, this planar source generates an initial plasma which is rich in toroidal field energy (typical poloidal currents are $100 \mathrm{kA}$ ) but poor in poloidal field energy. Spheromak formation involves conversion of toroidal magnetic flux into poloidal magnetic flux while conserving magnetic helicity (but not conserving magnetic energy).

\section{Sequence of Steps Intrinsic to Spheromak Formation}

Experimental observations of the steps intrinsic to spheromak formation have been made using singleframe and multiple-frame intensified CCD cameras having $\sim 10$ ns shutter speeds. By capturing the complex topological evolution occurring over a $\sim 10$ $20 \mu$ s interval these high-speed photos show that spheromak formation involves the following steps:

\section{Breakdown and Creation of a Number of Distinct, Arched, Filamentary, Plasma-Filled Flux Loops}

Just after breakdown, distinct filamentary loops are observed [4],[5] to span from the disk gas injector nozzles to the corresponding annulus nozzles; these loops form a pattern resembling the legs of a spider as shown in Fig.2. The filamentary nature of the spider legs and their association with gas injection is consistent with a recent model [6] shown 
schematically in Fig. 3. This model predicts that if the two ends of an axially non-uniform, bulging, open flux tube intercept particle sources at the wall (e.g., a gas puff valve as is the case here) then $\mathbf{J} \times \mathbf{B}$ forces will ingest plasma from the source and accelerate the injected plasma inwards along the flux tube towards a stagnation region. Frozen-in azimuthal magnetic flux convects with the ingested plasma and concentrates at the stagnation region, increasing the azimuthal magnetic field there. The increased azimuthal magnetic field pinches the flux tube causing it to develop an axially uniform cross-section and become filamentary. This is clearly seen in Fig. 2, where each spider leg has an axially uniform [5] (filamentary) cross-section, in marked contrast to the flaring crosssection of the initial vacuum magnetic field of Fig. 1. Because ingestion of plasma by the flux tube makes the flux tube filamentary, the availability of plasma to be ingested is a crucial requirement for this process. The loops in Fig. 2 are analogous to solar coronal loops and this ingestion-collimation model offers a likely explanation for why solar coronal loops are always observed [7] to be collimated.

\section{Merging of Loops to Form a Central Column}

The inboard portions of the arched, filamentary flux loops (i.e., the portions nearest the device geometric axis) merge to form a central column of bright plasma aligned along the device geometric axis; this is shown in Fig. 4. The merging occurs a few microseconds after breakdown. Magnetic probes indicate that the bright plasma is where the poloidal current is concentrated. This poloidal (axial) current flows along the central column from the center disk electrode and then returns to the outer annulus electrode forming an umbrella-like shroud surrounding the central column. The magnetic pressure of the toroidal magnetic field associated with the central column current tends to expand the umbrella shape. Symmetry of the structure is found to be dependent on the symmetry of the gas puffing, supporting the argument that the availability of gas to be ingested is a necessary condition for the formation of the observed magnetic structures. The poloidal flux at the coaxial disk and annulus electrodes is fixed because the experimental time scale is much faster than the resistive diffusion time scale in the disk and annulus electrodes.

\section{Jet-Like Expansion of Central Column}

MHD forces similar to those discussed in step 1, but with a different orientation, cause the central column to lengthen rapidly so that it can be considered to be an axial plasma jet as shown in Fig. 5. This mechanism is essentially similar to that sketched in Fig. 3, except here the system is single-ended rather than symmetrical about a midplane, i.e., the system is analogous to the right-hand half of Fig. 3 and the return current flows along the umbrella discussed in step 2. This plasma jet is also analogous to an astrophysical jet [2]. A magnetic probe, visible in the lower left of Fig. 5, has been used to measure magnetic field and current profiles. These measurements indicate that the current is concentrated in the bright region [3]. Furthermore, spectroscopic measurements of Stark broadening show that the particle density is also concentrated in the bright region. Measurements of the neutral density before breakdown show that negligible neutral particles were present before breakdown and so it can be concluded [5] that the observed high density plasma is convected by the jet and is not the result of ionization of previously existing in-place neutral gas. The velocity of the expanding jet is initially $\sim 20 \mathrm{~km} / \mathrm{s}$ (e.g. Fig. 4) and becomes as large as $\sim 60 \mathrm{~km} / \mathrm{s}$ (e.g. Fig.5) and depends on the gas species being used and on the poloidal current.

\section{Kink Instability of Central Column}

The central column jet can also be considered as being a lengthening, stabilized z-pinch. At a critical length, this jet with its embedded poloidal and toroidal fields satisfies the $q=1$ Kruskal-Shafranov kink instability condition. When this happens, the central column jet quickly becomes kink unstable and deforms into a well-defined helix [2] as shown in Fig. 6. These welldefined kinks are observed [2] over a limited range of the ratio of poloidal current to poloidal flux (lambda).

\section{Conversion of Toroidal Flux to Poloidal Flux by the Kink Instability}

The kink becomes so large that the helically deformed current channel effectively becomes the helical current of a solenoid. This creates new poloidal magnetic field and constitutes the dynamo-like conversion of toroidal to poloidal flux intrinsic to spheromak formation [3] (this conversion process was first postulated by Lindberg and Jacobsen [8]). Quantitative measurements of the magnetic fields in our experiment indicate that the magnitude of newly created poloidal flux is consistent with the notion that the new poloidal flux results from the winding of the initially axial current channel into a solenoid. Also, a simple model [3] shows that kinks are invariably paramagnetic, i.e., the helical sense of kinks is such as to always amplify 
the original magnetic field along which a force-free current flows.

\section{Detachment at High Lambda}

At values of lambda much higher than the values where well-defined kinks occur, it is seen that the central column quickly breaks off from the center electrode and flies off as a discrete separated object. This is shown in Fig. 7; it is conjectured that the bright region may be the core of a detached spheromak, but this has not yet been verified.

\section{DISCUSSION REGARDING CONVECTIVE EFFECTS}

Both the formation of the initial spider legs and the jetlike acceleration of the central column show that spheromak formation results from MHD pumping of plasma from the gas nozzles into the flux tube linking the nozzles. The filling up of the flux tube with plasma is thus a driven MHD process and not a diffusive transport process. Figure 8 shows that there is a larger pinch force at small $z$ (left in Fig.8) than at large $z$ (right in Fig.8). This pressure imbalance will create an on-axis pressure gradient that drives a jet-like flow along the axis to large $z$ (this is quite similar to squeezing the end of a toothpaste tube so that toothpaste squirts out from the mouth of the tube). However, the situation is more complicated [6] than just having an on-axis pressure gradient because jet flow is also driven off-axis, in this case by the axial gradient of the magnetic pressure associated with the toroidal field. This jet-like flow [6] convects both helicity and toroidal magnetic flux. Stagnation of the flow corresponds to the development of a negative divergence for the velocity (i.e., a convergence) as indicated in Fig.8. This stagnation leads to an accumulation of toroidal flux in the frame of the moving plasma at the stagnation region, increasing the toroidal magnetic field there and hence pinching the current channel. The plasma effectively acts like its own "zipper" and forms a lengthening self-collimating jet.

Doppler-shift measurements [5] of spectral lines using a $1 \mathrm{~m}$ spectrometer have corroborated the $\sim 20-60$ $\mathrm{km} / \mathrm{s}$ flow velocities observed using the high-speed photography. The combination of high speed photography, magnetic probe measurements, Stark broadening density measurements, and Doppler shift measurements together support the postulate that MHD forces can ingest large amounts of plasma from a wall source and that this ingestion has a profound effect on the shape of the magnetic configuration. This occurs because magnetic flux, being frozen into the ingested plasma, accumulates in tandem with the accumulation of mass. Stagnation of mass flows leads to concentration of embedded magnetic flux which tends to create filamentary flux tubes. At high currents these filamentary, mass-loaded flux tubes become kink unstable and can relax to form spheromak-like configurations.

We have recently arranged our gas puffing system to permit simultaneous injection of different gas species into the two ends of the flux tube linking the wall. In particular, nitrogen has been injected from the inner disk (cathode) nozzles while injecting neon from the outer annulus (anode) nozzles. By placing an optical filter in front of the camera lens, it has been possible to restrict the image to be mainly of the nitrogen coming from the inner disk electrode and thus visualize the flow of plasma along the spider leg.

Finally, we should point out that the images presented in Fig. 2 and Figs.4-7 do not come from a single plasma shot, but rather have been selected from different shots with different operating parameters and have been chosen to provide the clearest examples of each step in the sequence (e.g., the sharpest spider legs).

\section{POSSIBLE SEQUENCE FOR THE TOPOLOGICAL TRANSFORMATION FROM A JET INTO A SPHEROMAK}

The actual transformation from an attached jet-like structure into a detached spheromak has not yet been tracked. However, it is surmised that the sequence involves the jet kinking and then the kink detaching and compressing axially while expanding radially. First two "units" of helicity is injected into the configuration (by ramping up the poloidal current) to cause the configuration to evolve from the situation in Fig.9(a) to that in Fig.9(b). From a helicity conservation point of view, it is seen that a jet with a 720 degree twist (two complete turns) as in Fig. 9(b) will have two "units" of helicity. This can evolve in a helicity-conserving manner from the configuration in Fig. 9(b) to the configuration in Fig.9(f) which consists of two linked, untwisted flux tubes, a configuration that also has two units of helicity. The axial compression in Figs. 9(c) through (e) would result from the mutual attraction between the parallel currents in adjacent turns of the helical kink (like adjacent windings of a solenoid). The radial expansion from Fig. 9(b) to 9(c) would be due to the hoop force associated with the poloidal field interior to the helix (effectively the $\mathrm{B}_{\mathrm{z}}$ field of a solenoid). 


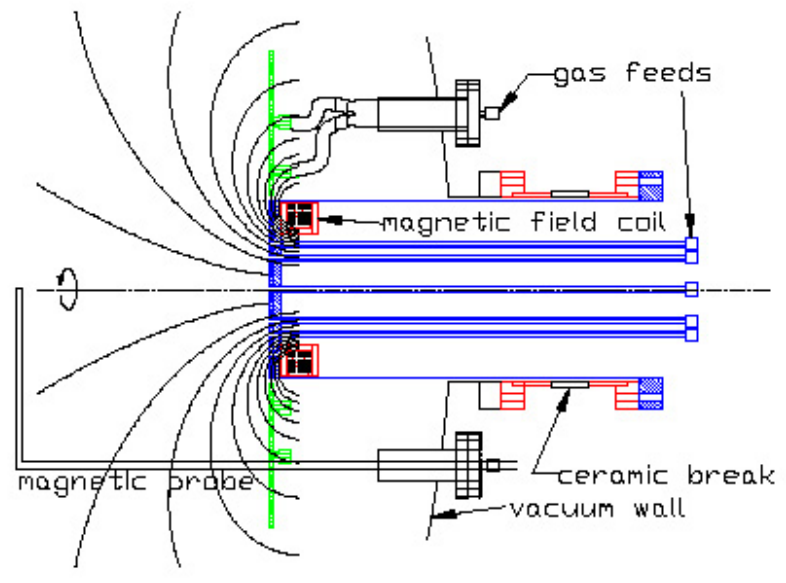

Figure 1 Coaxial electrostatic helicity source: inner (disk) electrode is $20 \mathrm{~cm}$ diameter, outer (annulus) electrode is 50 cm diameter. Linked flux is $1-2 \mathrm{mWb}$.

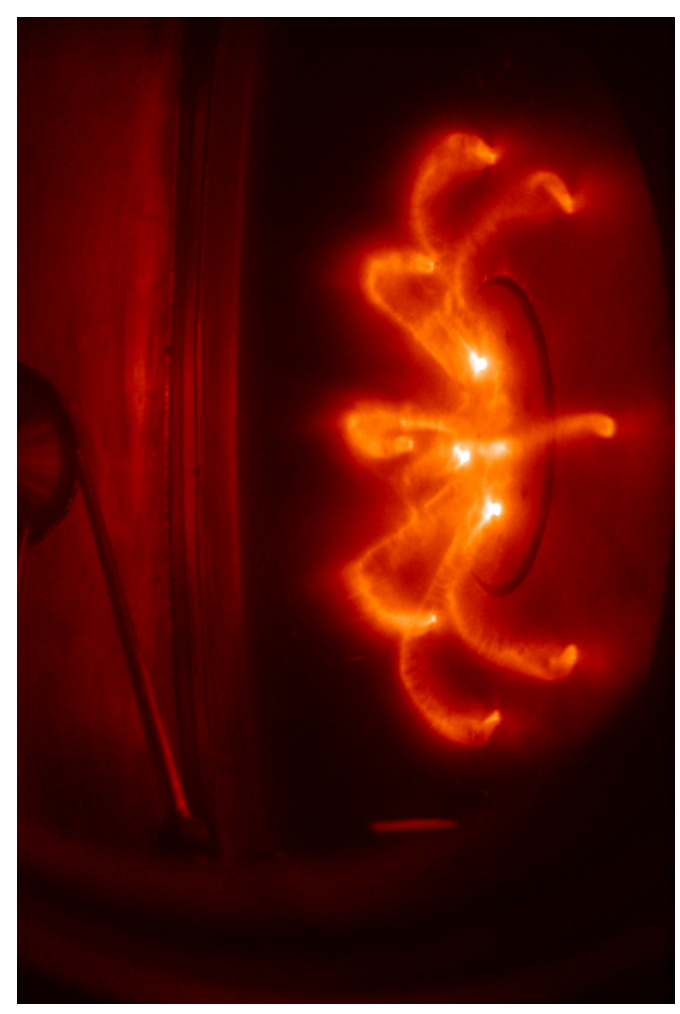

Figure 2 “Spider leg” pattern formed immediately after breakdown. \#4343: nitrogen-neon plasma, $1.2 \mathrm{mWb}, 3 \mu \mathrm{s}$.

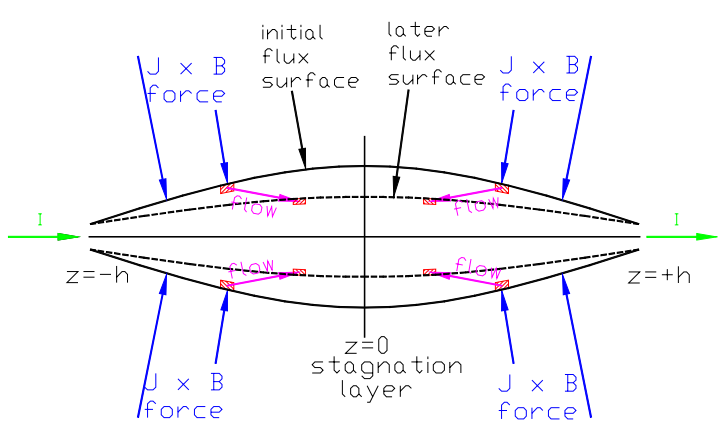

Figure 3 Ref. [6] model: The axial component of the $\mathbf{J} \times \mathbf{B}$ MHD force accelerates plasma from both ends to the middle. The radial component of the $\mathbf{J} \times \mathbf{B}$ MHD force causes higher on-axis pressure at both ends than at the middle; this higher pressure at the ends also accelerates plasma from both ends to the middle (i.e., to the $z=0$ layer).

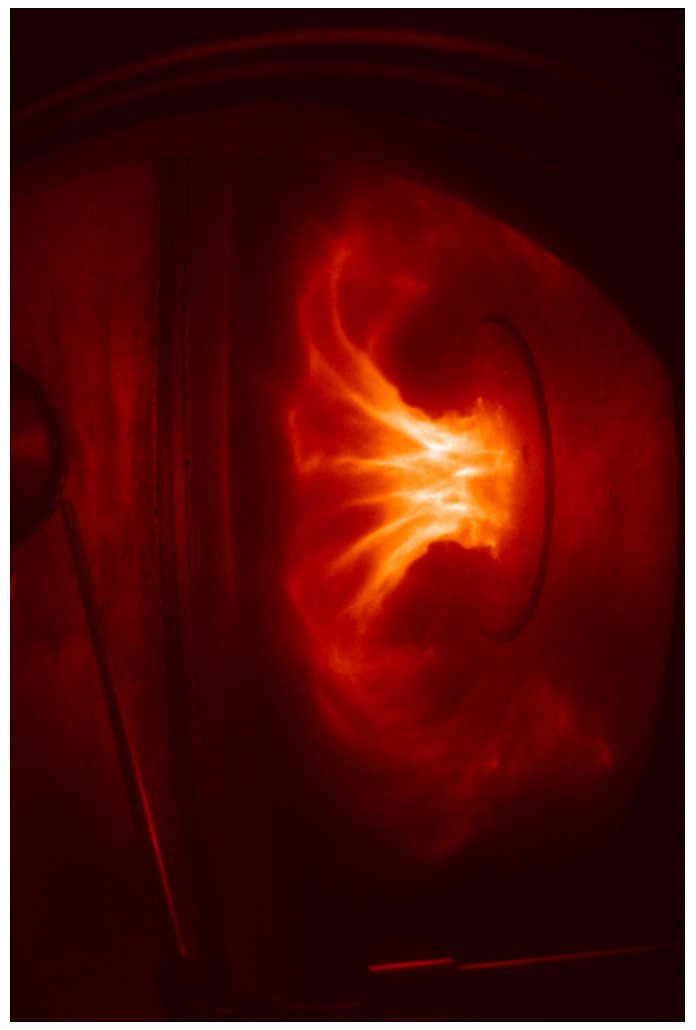

Figure 4 Merging of spider legs to form central column. Nitrogen plasm. \#4304: $1.2 \mathrm{mWb}, 6 \mu \mathrm{s}$. 


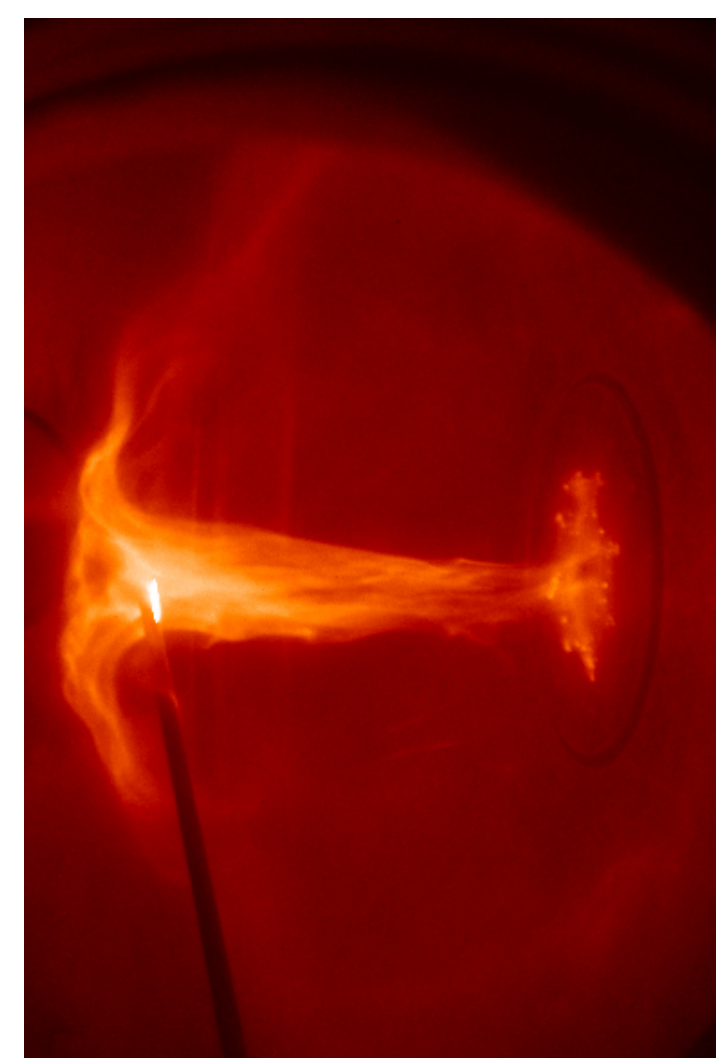

Figure 5 Jet-like expansion of central column. \#3444: 1.4 mWb, $10 \mu \mathrm{s}$.

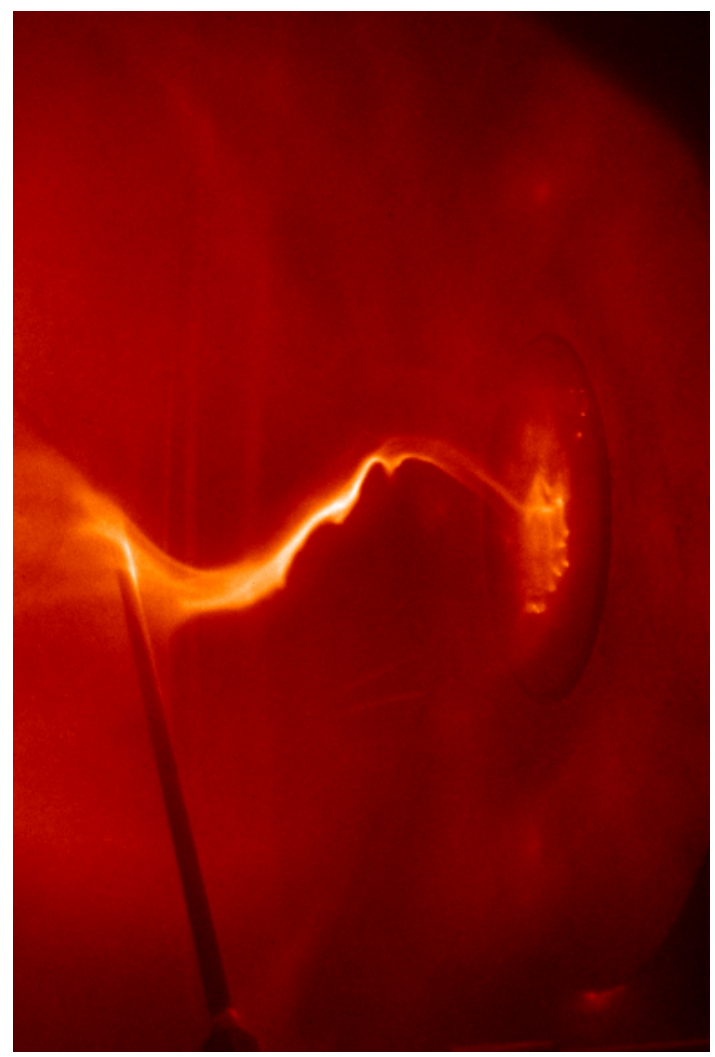

Figure 6 Kink instability occurs when jet attains critical length. \#3097: $1.3 \mathrm{mWb}, 6 \mu \mathrm{s}$.

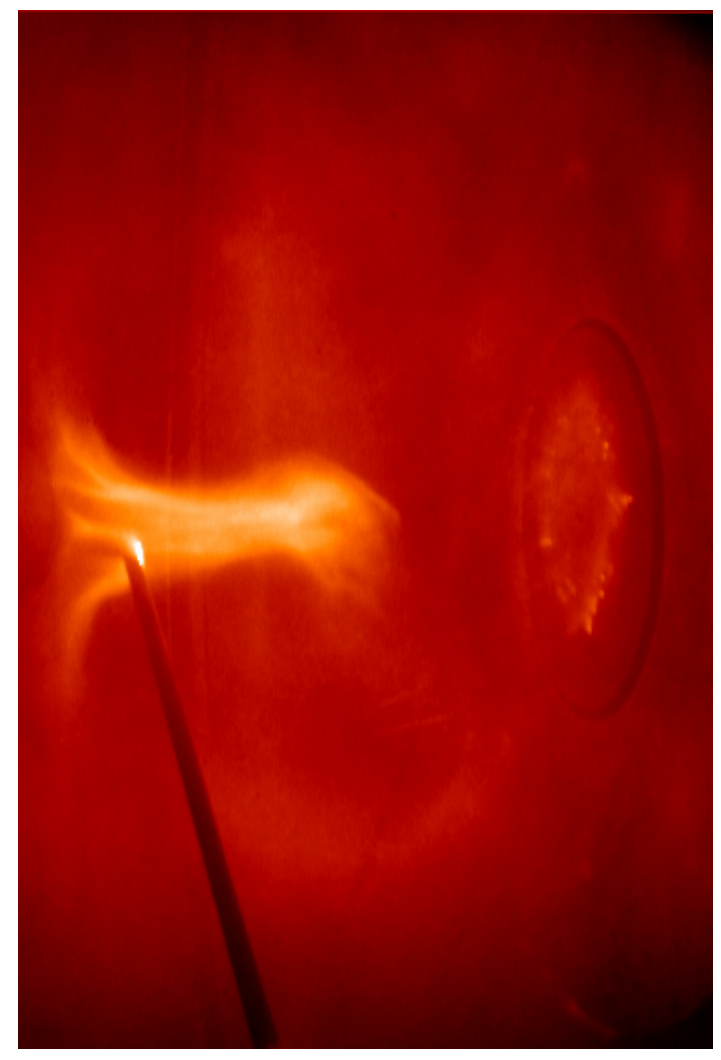

Figure 7 Detached hydrogen plasma at high lambda.

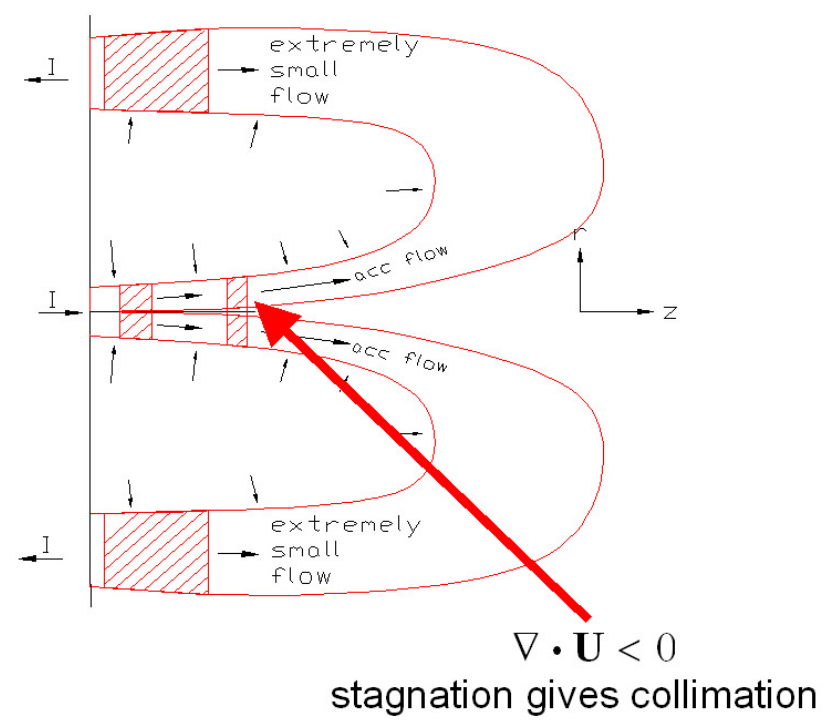

Figure 8 Sketch of poloidal field lines and mass (shaded regions) associated with planar coaxial helicity source (flow is from left to right here). The acceleration is mainly on the left where the forces are strongest and the flow velocity decreases on going from left to right. This decrease leads to a converging flow in the frame of the flow and hence collimation. 


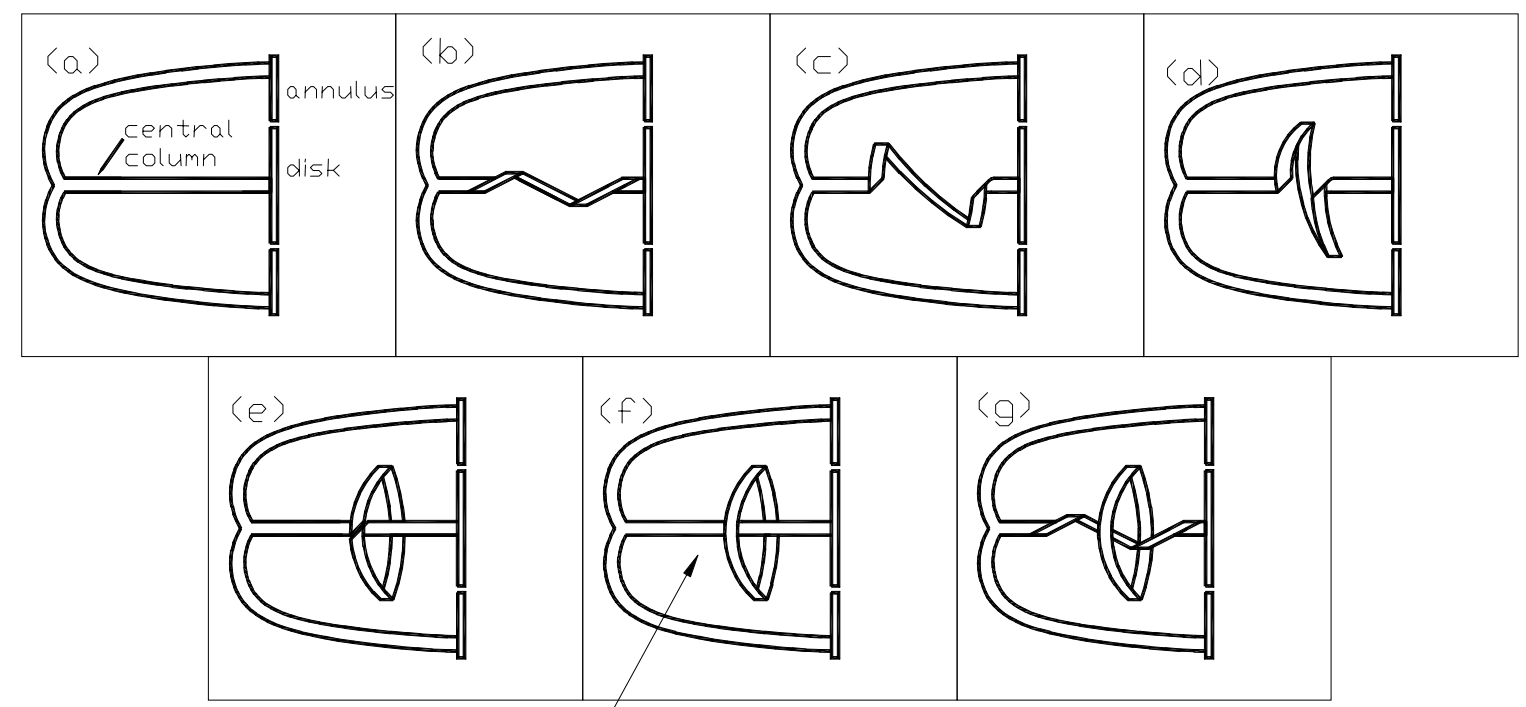

Figure 9 Possible 720-degree twist sequence for change in topology from jet to spheromak.

ACKNOWLEDGMENTS- supported by the United States Department of Energy.

[1] S. C. Hsu and P. M. Bellan, "Study of Magnetic Helicity Injection via Plasma Imaging Using a High-Speed Digital Camera”, IEEE Trans. Plasma Sci. 30, 10 (2002).

[2] S. C. Hsu and P. M. Bellan, "A laboratory plasma experiment for studying magnetic dynamics of accretion disks and jets”, Monthly Notices Royal Astronomical Society $\mathbf{3 3 4}$ (2002) 257.

[3] S. C. Hsu and P. M. Bellan, "Experimental identification of the kink instability as a poloidal flux amplification mechanism for coaxial gun spheromak formation”, Phys. Rev. Letters 90 (2003) article 215002.
[4] S. C. Hsu and P. M. Bellan, "On the jets, kinks, and spheromaks formed by a planar magnetized coaxial gun”, Phys. Plasmas 12, art. 032103 (2005)

[5] S. You, G. S. Yun, and P. M. Bellan, "Dynamic and stagnating plasma flow leading to magnetic-flux-tube collimation”, Phys. Rev. Lett. 95, art. 045002 (2005)

[6] P. M. Bellan, "Why current-carrying magnetic flux tubes gobble up plasma and become thin as a result”, Phys. Plasmas 10 (2003) 1999.

[7] J. A. Klimchuk, "Cross-sectional properties of coronal loops”, Solar Physics 193 (2000) 53.

[8] L. Lindberg and C. T. Jacobsen, "Studies of plasma expelled from a coaxial plasma gun", Phys. Fluids 7 (1964) S44. 